\title{
Universal band gap modulation by radial deformation in semiconductor single-walled carbon nanotubes
}

\author{
Kazume Nishidate ${ }^{1}$ and Masayuki Hasegawa ${ }^{2}$,* \\ ${ }^{1}$ Department of Electrical and Electronic Engineering, Iwate University, Morioka 020-8551, Japan \\ ${ }^{2}$ Department of Materials Science and Engineering, Iwate University, Morioka 020-8551, Japan
}

(Received 30 July 2008; published 4 November 2008)

\begin{abstract}
We report on a universal band gap modulation by radial deformation found for semiconductor single-walled carbon nanotubes (SWNTs). The plausible radial deformation of an individual SWNT under hydrostatic pressure is predicted using the method developed in the previous work [M. Hasegawa and K. Nishidate, Phys. Rev. B 74, 115401 (2006)]. It is found by ab initio electronic-structure calculations that the band gap of zigzag SWNTs is dictated by the shape of the high-curvature edge region of a deformed cross section perpendicular to the tube axis: If we let $R_{\min }$ be an averaged curvature radius in that region, the band gap at the $\Gamma$ point remains almost unchanged when $R_{\min } \geq 3.2 \AA$, and its closure occurs at $R_{\min } \approx 2.4 \AA$ irrespective of tube size and cross-sectional shape as a whole. It is also confirmed that the band gap closure is accompanied by the concentration, in the high-curvature region, of the lowest conduction state at the $\Gamma$ point. Possible implications of these results are discussed.
\end{abstract}

DOI: 10.1103/PhysRevB.78.195403

PACS number(s): 73.22.-f, 61.46.-w, 71.15.Mb, 73.63.Fg

\section{INTRODUCTION}

The electronic structures of single-walled carbon nanotubes (SWNTs) have been studied on the basis of how the band structure of the underlying graphitic sheet (graphene) is folded when the sheet is rolled up into a seamless cylinder. ${ }^{1}$ These results predicted on the basis of the zone-folding $(\mathrm{ZF})$ approximation were found to be modified by the curvatureinduced hybridization effect, ${ }^{2}$ and the extensive calculations, mostly based on the density functional theory (DFT), have been performed for a number of tubes. ${ }^{3-7}$ Summary of these earlier calculations for relatively small SWNTs is given in Ref. 5. According to these investigations, all the armchair $(n, n)$ SWNTs are metallic, and zigzag $(n, 0)$ SWNTs with $n>6$ are semiconducting. While, chiral $(n, m)$ SWNTs are metallic if the chiral indices satisfy the condition $n-m=3 i$, where $i$ is an integer, in accordance with the ZF approximation, and otherwise they are small-gap semiconductors. These theoretical predictions have been evidenced for particular SWNTs by the scanning tunneling microscope (STM) measurements, ${ }^{8}$ which can simultaneously probe the atomic structures and electronic properties. In recent years, the structural and chemical modifications of carbon nanotubes have attracted increasing attention with a motivation of finding possible technological applications. ${ }^{9}$ These modifications include various types of structural distortion and adsorptions of foreign atoms or molecules. In particular, individual SWNTs are prone to a significant cross-sectional deformation under an external stress as demonstrated by experiments ${ }^{10,11}$ and theoretical calculations. ${ }^{12-16}$ The radial deformation of small SWNTs whose diameters are smaller than $\sim 25 \AA$ is reversible, ${ }^{12,16}$ while the deformation of larger tubes could be irreversible and a collapsed state is metastable or even absolutely stable. ${ }^{11,16}$ Of particular interest is that the band gap of a semiconductor SWNT varies with radial deformation and eventually closes at a critical deformation. ${ }^{17-23}$ This electronic-structure transformation has an important implication for device applications. How- ever, this phenomenon has not been fully investigated partly because of the difficulty in systematically treating radial deformation under an external stress. In the present work we are concerned with the individual zigzag $(3 i \pm 1,0)$ SWNTs with relatively large band gaps and report on a universal band gap modulation by radial deformation found for these tubes. The plausible radial deformation under hydrostatic pressure is predicted using the method we have developed in the previous work, ${ }^{16}$ which enables us to predict deformations of an individual SWNT quite easily.

In Sec. II we summarize the method used to predict radial deformation and show the results for particular SWNTs of interest. In Sec. III we present $a b$ initio DFT electronicstructure calculations for the radially deformed SWNTs and discuss on these results. The final section (Sec. IV) is devoted to the conclusions.

\section{RADIAL DEFORMATION}

It has been confirmed by DFT and molecular-mechanics calculations that the strain energy, defined as the energy increase due to the curvature effect, of undeformed (circular) SWNTs is well represented as $E_{\text {strain }}=\alpha / R_{0}^{2}$, where $R_{0}$ is the tube radius and $\alpha$ is a constant insensitive to $R_{0}$ and chirality of a tube. This result is in accordance with the classical result for the continuum elastic shell and has been extended to radially deformed SWNTs. ${ }^{16}$ Also included in this extension are the effects not taken into account in the elastic shell model. One of these effects is the interwall interaction that becomes significant when the radial deformation is large enough to cause overlapping of the electron distribution in between opposing walls. Another is the van der Waals (vdW) interaction certainly missed in the standard DFT approximations such as the local density approximation (LDA) and generalized gradient approximation (GGA). The vdW contribution was treated by a semiempirical method, which has originally been developed to describe the interlayer binding of graphite. ${ }^{24}$ 


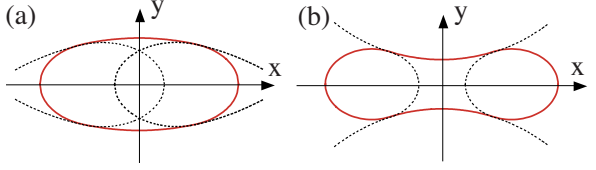

FIG. 1. (Color online) Model cross-sectional shape of a radially deformed SWNT. The $z$ axis is taken to be in the direction of the tube axis. The circumference of each shape consists of two ellipsoids connected by two circles on the top and bottom. Each shape, (a) oval or (b) peanut, transforms into another through a racetracklike shape, in which circular parts are parallel, straight lines (i.e., circles with infinite curvature radii).

The second step toward the prediction of radial deformations of a SWNT under an external stress is to assume a plausible cross-sectional shape. Following the previous work, ${ }^{16}$ we assumed a model shape whose circumference consists of two ellipsoids smoothly connected by two circulars, as illustrated in Fig. 1. This model shape is characterized by four parameters, and one of them can be eliminated by assuming that the perimeter of a deformed cross section remains unchanged and is equal to $2 \pi R_{0}$, where $R_{0}$ is the circular radius of the undeformed tube. Then, for a given fractional change (decrease) in cross-sectional area $S$,

$$
X=1-S / S_{0}\left(S_{0}=\pi R_{0}^{2}\right),
$$

remaining shape parameters are determined so as to minimize the strain energy. Here, we also assumed that no deformation occurs in the axial direction, which implies that $X$ also represents the fractional change in volume of a deformed tube and provides a useful parameter in treating radial deformation. The cross-sectional shape optimized in this way for a given $X$ is expected to be, though approximately, consistent with the applied hydrostatic pressure. In fact, the model cross-sectional shapes determined by this method are quite similar to those predicted by constant-pressure molecular-dynamics simulations using empirical atom-atom interaction potentials. ${ }^{13-15}$

We considered zigzag $(8,0),(10,0)$, and $(14,0)$ SWNTs with relatively large band gaps. Figure 2 shows the optimized deformation energy $\Delta E$, defined as the strain energy per atom relative to the undeformed circular tube, as a func-
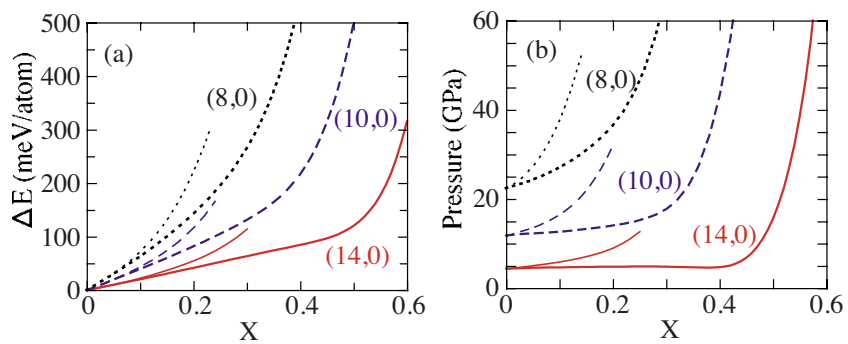

FIG. 2. (Color online) Calculated deformation energies of the $(8,0)$ (dotted line), (10,0) (dashed line), and $(14,0)$ (solid line) SWNTs, and the hydrostatic pressures necessary to deform these tubes as functions of the deformation parameter $X$ defined by Eq. (1). The thin line for each tube shows the result when an elliptic cross section is assumed. tion of $X$ and the corresponding hydrostatic pressure necessary to deform these tubes. This pressure is given by

$$
P=-2 \pi R_{0} \rho\left(\frac{\partial \Delta E}{\partial V}\right)=\frac{2 \rho}{R_{0}}\left(\frac{\partial \Delta E}{\partial X}\right),
$$

where $\rho$ is the atomic number density of the underlying graphene and $V$ is the volume of the tube per unit length along the tube axis. The results in Fig. 2 indicate that the radial deformations of all those tubes are reversible up to large deformation; i.e., flattened tubes are inflated on pressure release. The minimum pressure $P_{0}$ required for the deformation to occur is given by the above pressure at $X=0$ and is nothing but the circular-to-oval transition pressure. ${ }^{15,16}$ Since the deformation energy $\Delta E$ in the small deformation regime scales as $R_{0}^{-2}$ in accordance with the classical result, the transition pressure $P_{0}$ is closely proportional to the inverse cube of tube radius and given by $22.4,11.8$, and 4.4 GPa for the $(8,0),(10,0)$, and $(14,0)$ SWNTs, respectively. We also note that an elliptic shape often assumed for a deformed cross section ${ }^{22}$ is unlikely because the optimized $\Delta E$ for such a shape is much higher than that obtained for a more flexible shape assumed in our calculations [Fig. 1(a)]. If we assume an elliptic cross section the pressure required to deform these tubes also increases more rapidly with increasing deformation [Fig. 2(b)]. We find that once the $(14,0)$ SWNT, and larger tubes ${ }^{16}$ have started to deform at $P_{0}$, the deformation spontaneously proceeds up to a flattened shape with $X>0.4$, which is peanutlike rather than oval-like.

At this stage we note a general feature of the radial deformation of SWNTs. ${ }^{16}$ A circular SWNT remains undeformed and simply shrinks below a critical pressure, at which radial deformation starts to occur, in accordance with the classical buckling theory. This critical pressure is nothing but $P_{0}$ defined in the above. Following the previous work, ${ }^{16}$ the tube radius $R_{0}$ at $P=P_{0}$ was assumed to be the same as that under zero pressure and equal to that obtained by rolling up the graphene sheet. In fact, the shrinking of a circular tube is very small because its radial bulk modulus is very high compared to $P_{0}$.

\section{ELECTRONIC STRUCTURES OF DEFORMED TUBES}

To calculate electronic structure of a deformed SWNT we assumed an atomic configuration obtained by the conformal mapping of carbon atoms on the deformed tube surface determined on the basis of the continuum model. This configuration has also been used to test the validity of the simple method in calculating the deformation energy. ${ }^{16}$ The DFT electronic-structure calculations were performed using the Vienna Ab Initio Simulation Program (VASP) code ${ }^{25}$ In these calculations we employed the LDA with the exchangecorrelation energy functional of Ceperley and Alde, ${ }^{26}$ as parametrized by Perdew and Zunge, ${ }^{27}$ and the projectoraugmented wave (PAW) method. ${ }^{28}$ The $k$-space integration was made using the Monkhorst-Pack method ${ }^{29}$ with the special points in the irreducible Brillouin zone generated from $1 \times 1 \times 8$ mesh. We used a large supercell in the plane perpendicular to the tube axis to simulate an isolated SWNT, 

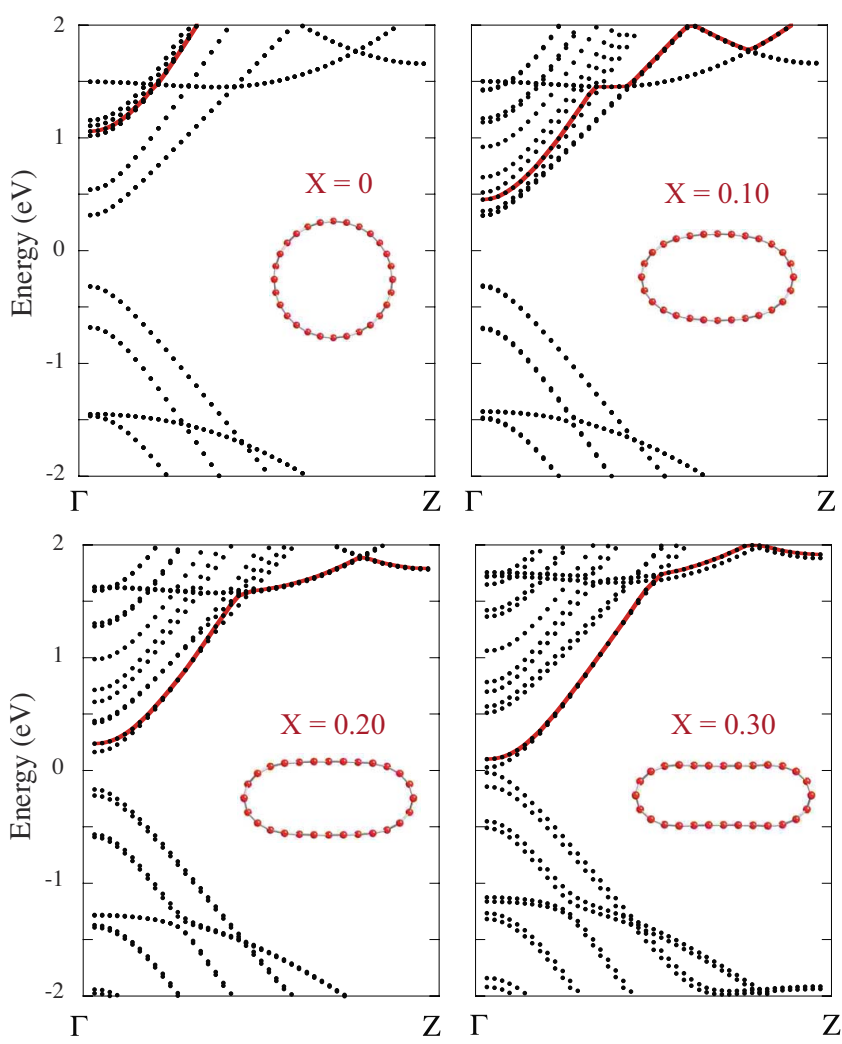

FIG. 3. (Color online) Energy-band structures of the circular and radially deformed $(14,0)$ SWNT along the direction in the Brillouin zone parallel to the tube axis. The Fermi energy is taken to be zero. The solid lines near the $\Gamma$ point show the singlet state.

and the cutoff energy of $500 \mathrm{eV}$ was used in the plane-wave expansion.

Figure 3 illustrates, as an example, the energy-band structures of the $(14,0)$ SWNT along the direction in the Brillouin zone parallel to the tube axis. The band gaps $E_{g}$ at the $\Gamma$ point of the undeformed circular tubes $(X=0)$ were found to be $0.553,0.903$, and $0.633 \mathrm{eV}$ for the $(8,0),(10,0)$, and $(14,0)$ SWNTs, respectively. These values are in the range of the previous calculations in either $\mathrm{LDA}$ or $\mathrm{GGA},{ }^{2-7}$ i.e., $0.43-0.73 \mathrm{eV}$ for the $(8,0), 0.76-0.91 \mathrm{eV}$ for the $(10,0)$, and $0.63-0.90 \mathrm{eV}$ for the $(14,0)$ SWNTs. We note that the details other than DFT approximations such as the geometrical optimization have some influence on the electronic structures of these SWNTs. We actually find some, but insignificant, differences between our results for $E_{g}$ and those of Zolyomi and Kürti, ${ }^{5}$ which are $E_{g}=0.59,0.77$, and $0.72 \mathrm{eV}$ for the $(8,0),(10,0)$, and $(14,0)$ SWNTs, respectively. These results have been obtained by similar LDA calculations with geometrical optimization. Recently, Kamal and Chakrabarti ${ }^{7}$ suggested the need of complete geometrical optimization and all-electron calculations for very small tubes, typically smaller than the $(6,0)$ tube. Such computational details may not have a crucial influence on the electronic structures of the relatively large tubes under consideration. As the radial deformation increases, the band gap becomes narrower and eventually closes at a critical deformation depending on the tube size as already found for the relatively small zigzag SWNTs. ${ }^{17-23}$ These behaviors can be seen more clearly in
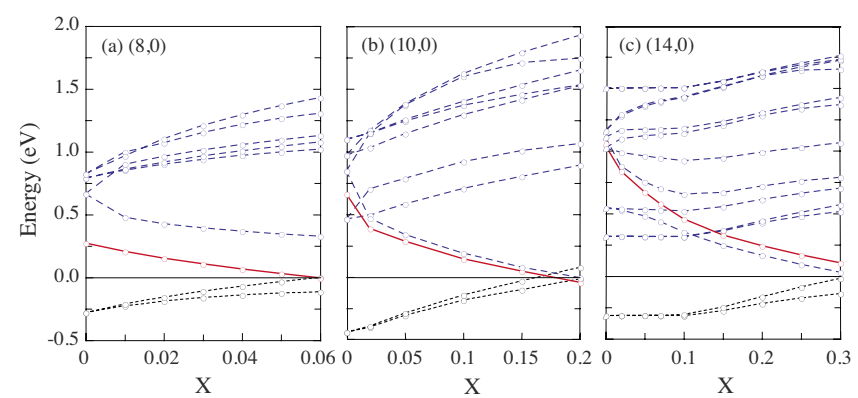

FIG. 4. (Color online) Variations in the calculated band energies at the $\Gamma$ point with the deformation parameter $X$ defined by Eq. (1). The Fermi energy is taken to be zero. The solid line for each tube shows the singlet state, and all the other states-both in the valence (dotted lines) and conduction (dashed lines) bands-are doubly degenerate in the circular $(X=0)$ tubes and are split as $X$ increases.

Fig. 4, where the variations in the band energies at the $\Gamma$ point are shown as a function of the deformation parameter $X$ defined by Eq. (1). For the $(8,0)$ tube, the singlet state is the lowest conduction band and is responsible for the band gap narrowing and closure with increasing radial deformation. These band gap behaviors are essentially the same as those found by Gülseren et al. ${ }^{22}$ for the $(7,0)$ and $(8,0)$ SWNTs by assuming an elliptic cross section and may be interpreted as the effect of the enhanced $\pi^{*}-\sigma^{*}$ hybridization. ${ }^{17,20}$ For the $(10,0)$ tube, the singlet state originally above the doublydegenerate state lowers (in energy) with increasing deformation and crosses a low-lying conduction state, and after crossing, the role of the singlet state is similar to that in the $(8,0)$ tube: At the first stage without crossing $(X<0.015)$, the band gap remains almost unchanged because the lowest conduction state and the highest valence state vary very little with $X$ in that range. This behavior has been found, but not clarified, by Mazzoni and Chacham ${ }^{18}$ for the same $(10,0)$ tube with a racetracklike cross section, which consists of two circular edge part connected by two straight lines. For larger zigzag $(3 i \pm 1,0)$ SWNTs, this characteristic behavior for the $(10,0)$ tube is more pronounced as demonstrated for the $(14,0)$ SWNT. For this relatively large tube, the effect of the radial deformation on the conduction-band minimum (CBM) and the valence-band maximum (VBM), both at the $\Gamma$ point, are minimal, and the band gap remains almost unchanged up to a large deformation $(X \approx 0.10)$ [Figs. 3 and $4(\mathrm{c})$ ]. In this case, the lowering of the split band, originally $(X=0)$ the second lowest in the conduction bands, crosses the lowest conduction state at $X \approx 0.10$, and after crossing the lowered state is responsible for the band gap narrowing and closure. We find that, for all these $(3 i \pm 1,0)$ SWNTs, the lowering of the singlet state with increasing deformation is accompanied by that of the split band, which is doubly degenerate in the circular $(X=0)$ tube and lies just above the singlet state.

The band gaps at the $\Gamma$ point are summarized in Fig. 5(a) as a function of $X$, and we find that the critical deformation corresponding to band gap closure is larger for larger tubes. But we also find that the band gap narrowing and closure are dictated by the local shape with the highest curvature as illustrated in Fig. 5(b), which shows the variations in the band gaps as a function of the mean curvature radius averaged over the high-curvature region, 

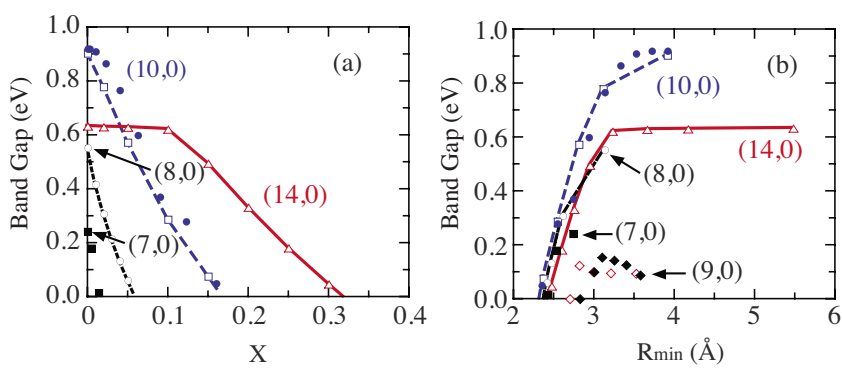

FIG. 5. (Color online) Variations in the band gaps of the deformed $(8,0)$ (dotted line), $(10,0)$ (dashed line), and $(14,0)$ (solid line) SWNTs as functions of $X$ and $R_{\min }$ defined by Eqs. (1) and (3), respectively. Also included are the previous results for the $(7,0)$ tube (closed squares, from Ref. 22), (9,0) tube (closed diamonds, from Ref. 17; open diamonds, from Ref. 22), and $(10,0)$ tube (closed circles, from Ref. 18).

$$
R_{\min }=\frac{1}{2 \Delta s} \int_{-\Delta s}^{\Delta s} R(s) d s
$$

where $R(s)$ is the local curvature radius along the circumference and takes its minimum value at $s=0$, the edge point of a flattened tube. Here, we used the value of $\Delta s=2.46 \AA$, which is the distance between the carbon atoms along the circumference in case of zigzag tubes and is nothing but the lattice constant of graphene. This integration range was chosen because the local curvature is appropriately defined over the range in which two neighboring atoms are involved. If the high-curvature edge region of a flattened tube is circular as it is in the model used by Park et al. ${ }^{17}$ and Mazzoni and Chacham, ${ }^{18} R_{\text {min }}$ is simply given by that radius. In the present model that part is elliptic, and the model shape used by Gülseren et $a l^{22}$ is also an ellipsoid as a whole. The results in Fig. 5(b) indicate that the band gap remains unchanged when $R_{\text {min }}>3.2 \AA$ and closes at $R_{\text {min }} \approx 2.4 \AA$ irrespective of tube size and cross-sectional shape as a whole. This universal feature is consistent with that undeformed zigzag $(3 i \pm 1,0)$ SWNTs with small diameter are metallic. We also note that the results of Gülseren et al. ${ }^{22}$ are based on the electronic-structure calculations with fully optimized tube structures under the constraint that the cross-sectional shape is fixed to be elliptic. Their results for the $(7,0)$ and $(8,0)$ tubes also support the universal feature as exemplified for the $(7,0)$ tube in Fig. 5(b), suggesting that geometrical optimization does not much affect the general feature. A similar analysis can be made in terms of the aperture angle formed by three neighboring atoms along the same circumference of a deformed tube. This angle takes its minimum value, $\theta_{\min }$, when the central atom is on the edge point, and we find that the band gap closure occurs at $\theta_{\min } \approx 110^{\circ}$.

Figure 6 illustrates the state density (SD) due to the bandedge states at the $\Gamma$ point of the $(14,0)$ SWNT. The SD is nothing but an actual charge density in case of the occupied conduction state. In the small deformation regime with $X$ $<0.10$, the SD due to the CBM state is almost symmetric on the inner and outer sides of the tube. For large deformations with $X>0.10$, the SD due to the rapidly lowering CBM state [Figs. 3 and 4(c)] becomes asymmetric with lager concentra-

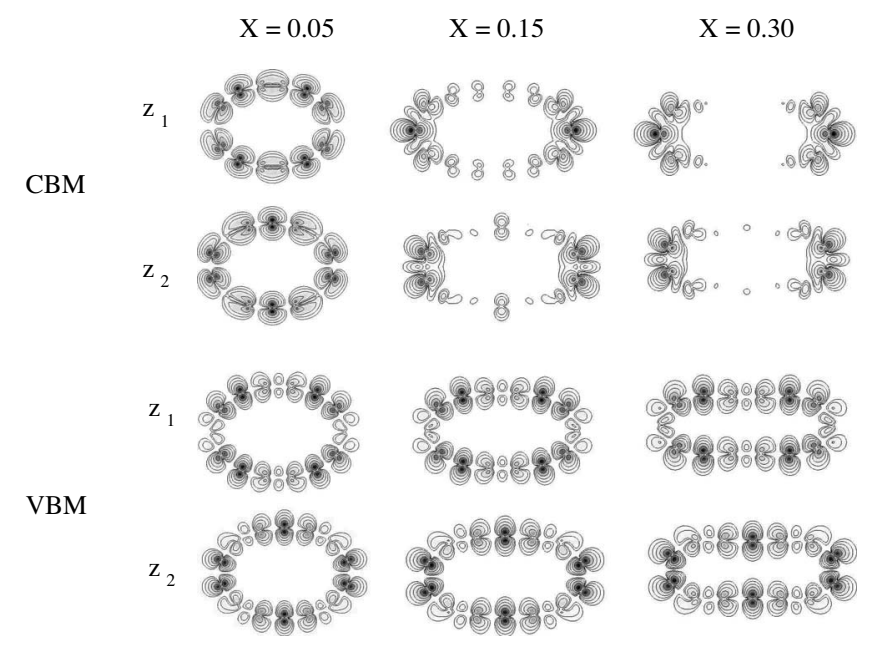

FIG. 6. State density (charge density in case of the VBM) due to the band-edge states at the $\Gamma$ point for the $(14,0)$ SWNT in the atomic planes at $z=z_{1}$ and $z=z_{2}$, both perpendicular to the tube axis and corresponding to a zigzag atomic-chain configuration in the tube. These planes are equivalent in the circular $(X=0)$ tube

tion on the outer side. This SD also tends to concentrate in the high-curvature edge region as the tube is flattened. On the other hand, the SD due to the VBM state is almost symmetric on both sides and shows no appreciable concentration in a particular region. These behaviors of the SD in the $(14,0)$ tube are similar to those found by Park et al. ${ }^{17}$ and Gülseren et ll. $^{22}$ for the small-gap $(9,0)$ SWNT. The results in Fig. 6 imply that once the tube is metallized by flattening the electronic conduction along the tube occurs through the high-curvature edge regions. In other words, a pair of parallel nanowires is formed in a single tube. Recently, Giusca $e t$ $a l .{ }^{30}$ made challenging STM measurements on the flattened tube, identified as the armchair $(21,21)$ SWNT with diameter $\approx 28 \AA$. They observed in these experiments that the flat region shows a metallic nature with a finite density of states at the Fermi level, while the band gap opens in the edge region at either side of the flattened tube, indicative of a semiconducting nature. These observations for the armchair SWNT are in marked contrast to the implications found for the zigzag SWNTs, which shows the reversed natures of the flat and edge regions. This difference between the armchair and zigzag SWNTs may arise from the different natures of the band-edge state near the Fermi level, but the microscopic picture is not resolved.

\section{CONCLUSIONS}

We have elucidated the band gap modulation by radial deformation in the zigzag SWNTs by assuming a realistic cross-sectional shape expected under hydrostatic pressure. In particular, we found a universal feature indicating that the narrowing and closure of band gaps are dictated by the local shape with high curvature along the circumference of a deformed cross section. If we let $R_{\text {min }}$ be an average curvature radius in that region, band gap remains unchanged when $R_{\min }>3.2 \AA$, and its closure occurs at $R_{\min } \approx 2.4 \AA$ irrespective of tube size and cross-sectional shape as a whole. It 
was also confirmed that the band gap closure of collapsed zigzag SWNTs is induced by the concentration of the lowest conduction state in the edge region. Very recently, Barboza et $a l{ }^{31}$ actually probed via electric force microscopy the deformation-induced semiconductor-metal transition in rather large chiral $(18,4)$ SWNT deformed under the tip of an atomic force microscope (AFM). They have also reinforced these observations by the $a b$ initio DFT electronic-structure calculations for this tube and zigzag $(20,0)$ SWNT of similar size by assuming a racetracklike cross-sectional shape. ${ }^{17,18}$ Their results for the band gap behavior in the $(20,0)$ SWNT are consistent with our results, although the band gap closure is predicted to occur at $R_{\min } \approx 2.75 \AA$, somewhat larger than the universal value, if the edge part of an assumed cross section is really spherical.

In the present work we considered only the extended radial deformation along the tube axis, but a localized deformation may be a more realistic situation encountered in device applications. ${ }^{20}$ If the deformation is not localized in a very small region, the present results may be valid as suggested by the recent experiments, ${ }^{31}$ in which the estimated radius of an AFM tip used to deform a SWNT is much larger than the tube radius, implying that the radial deformation is substantially extended in the axial direction. The present method could be extended to such a localized deformation with some complications in the structural optimization and substantial increase in computational cost. We also note that the radial deformation, either extended or localized, of small SWNTs is elastic, implying that the tubes flattened by an external stress are not stabilized but inflated on removing a stress. This brings about a hindrance to some device applications without practical measure to freeze a deformation. We have no such difficulty for large SWNTs, typically larger than $\sim 25 \AA$ in diameter, ${ }^{16}$ as we have noted earlier, and such a large tube may be a promising candidate in deformation-related applications. We finally notice a recent work by Chen et al., ${ }^{32}$ who investigated the electronic structure of SWNT bundles under uniaxial stress. The present results for individual SWNTs may be useful in understanding their results, which reflect superimposed effects due to the deformation of an individual tube and tube-tube interaction.

\section{ACKNOWLEDGMENTS}

This work was supported by the Iketani Foundation and a Grant-in-Aid for Scientific Research from the JSPS under Grant No. 20540394. We acknowledge the permission to use VESTA in graphics. The computations in this work were performed using the facilities of the Information Processing Center, Iwate University, and the Supercomputer Center at the Institute for Solid State Physics, University of Tokyo.
*Corresponding author; hasegawa@iwate-u.ac.jp

${ }^{1}$ N. Hamada, S. Sawada, and A. Oshiyama, Phys. Rev. Lett. 68, 1579 (1992); R. Saito, M. Fujita, G. Dresselhaus, and M. S. Dresselhaus, Appl. Phys. Lett. 60, 2204 (1992); J. Mintmire, B. I. Dunlap, and C. T. White, Phys. Rev. Lett. 68, 631 (1992).

${ }^{2}$ X. Blase, L. X. Benedict, E. L. Shirley, and S. G. Louie, Phys. Rev. Lett. 72, 1878 (1994).

${ }^{3}$ O. Gülseren, T. Yildirim, and S. Ciraci, Phys. Rev. B 65, 153405 (2002).

${ }^{4}$ G. Sun, J. Kürti, M. Kertesz, and R. H. Baughman, J. Phys. Chem. B 107, 6924 (2003).

${ }^{5}$ V. Zolyomi and J. Kürti, Phys. Rev. B 70, 085403 (2004).

${ }^{6}$ B. Kozinsky and N. Marzari, Phys. Rev. Lett. 96, 166801 (2006).

${ }^{7}$ C. Kamal and A. Chakrabarti, Phys. Rev. B 76, 075113 (2007).

${ }^{8}$ T. W. Odom, J. L. Huang, P. Kim, and C. M. Lieber, Nature (London) 391, 62 (1998); J. W. G. Wildöer, L. C. Venema, A. G. Rinzler, R. E. Smalley, and C. Dekker, ibid. 391, 59 (1998).

${ }^{9}$ R. H. Baughman, A. A. Zakhidov, and W. A. de Heer, Science 297, 787 (2002); S. Ciraci, S. Dag, T. Yildirim, O. Gülseren, and R. T. Senger, J. Phys.: Condens. Matter 16, R901 (2004); M. P. Anantram and F. Leonard, Rep. Prog. Phys. 69, 507 (2006).

${ }^{10}$ N. G. Chopra, L. X. Benedict, V. H. Crespi, M. L. Cohen, S. G. Louie, and A. Zettl, Nature (London) 377, 135 (1995).

${ }^{11}$ L. X. Benedict, V. H. Crespi, N. G. Chopra, A. Zettl, M. L. Cohen, and S. G. Louie, Chem. Phys. Lett. 286, 490 (1998).

${ }^{12}$ P. E. Lammert, P. Zhang, and V. H. Crespi, Phys. Rev. Lett. 84, 2453 (2000).

${ }^{13}$ D. Y. Sun, D. J. Shu, M. Ji, F. Liu, M. Wang, and X. G. Gong,
Phys. Rev. B 70, 165417 (2004).

${ }^{14}$ R. B. Capaz, C. D. Spataru, P. Tangney, M. L. Cohen, and S. G. Louie, Phys. Status Solidi B 241, 3352 (2004).

${ }^{15}$ J. Zang, A. Treibergs, Y. Han, and F. Liu, Phys. Rev. Lett. 92, 105501 (2004).

${ }^{16}$ M. Hasegawa and K. Nishidate, Phys. Rev. B 74, 115401 (2006).

${ }^{17}$ C. J. Park, Y. H. Kim, and K. J. Chang, Phys. Rev. B 60, 10656 (1999).

${ }^{18}$ M. S. C. Mazzoni and H. Chacham, Appl. Phys. Lett. 76, 1561 (2000).

${ }^{19}$ P. E. Lammert, P. Zhang, and V. H. Crespi, Phys. Rev. Lett. 84, 2453 (2000).

${ }^{20}$ Ç. Kiliç, S. Ciraci, O. Gülseren, and T. Yildirim, Phys. Rev. B 62, R16345 (2000).

${ }^{21}$ H. S. Sim, C. J. Park, and K. J. Chang, Phys. Rev. B 63, 073402 (2001).

${ }^{22}$ O. Gülseren, T. Yildirim, S. Ciraci, and Ç. Kiliç, Phys. Rev. B 65, 155410 (2002).

${ }^{23}$ J. Q. Lu, J. Wu, W. Duan, F. Liu, B. F. Zhu, and B. L. Gu, Phys. Rev. Lett. 90, 156601 (2003).

${ }^{24}$ M. Hasegawa and K. Nishidate, Phys. Rev. B 70, 205431 (2004); M. Hasegawa, K. Nishidate, and H. Iyetomi, ibid. 76, 115424 (2007)

${ }^{25}$ G. Kresse and J. Furthmüller, Phys. Rev. B 54, 11169 (1996); Comput. Mater. Sci. 6, 15 (1996).

${ }^{26}$ D. M. Ceperley and B. J. Alder, Phys. Rev. Lett. 45, 566 (1980).

${ }^{27}$ J. P. Perdew and A. Zunger, Phys. Rev. B 23, 5048 (1981).

${ }^{28}$ G. Kresse and D. Joubert, Phys. Rev. B 59, 1758 (1999). 
${ }^{29}$ H. J. Monkhorst and J. D. Pack, Phys. Rev. B 13, 5188 (1976).

${ }^{30}$ C. E. Giusca, Y. Tison, and S. R. P. Silva, Phys. Rev. B 76, 035429 (2007).

${ }^{31}$ A. P. M. Barboza, A. P. Gomes, B. S. Archanjo, P. T. Araujo, A.
Jorio, A. S. Ferlauto, M. S. C. Mazzoni, H. Chacham, and B. R. A. Neves, Phys. Rev. Lett. 100, 256804 (2008).

${ }^{32}$ D. Chen, T. Sasaki, J. Tang, and L. C. Qin, Phys. Rev. B 77, 125412 (2008). 